\title{
Particle Physics Outreach as a Strategic Pillar for Society: A Report from IPPOG
}

\author{
Sascha Mehlhase \\ on behalf of the IPPOG Collaboration \\ Ludwig-Maximilians-Universität München / ATLAS Collaboration \\ E-mail: sascha.mehlhase@cern.ch
}

\begin{abstract}
The International Particle Physics Outreach Group (IPPOG) is a network of scientists, science educators and communication specialists working across the globe in informal science education and outreach for particle physics. The IPPOG Collaboration comprises 30 members: 24 countries, 5 experiments and CERN as an international laboratory. We will report on current activities, including the continued growth and development of the International Masterclasses on Particle Physics programme, the outreach resource database, support for exhibitions and activities at public events and festivals, and topical programmes targeting young and diverse audiences. We will also discuss the strategic necessity of education and outreach to gain long-term public and stakeholder support for the field of particle physics, both in the context of the European Particle Physics Strategic Update (EPPSU), and in general for our society.
\end{abstract}

7th Annual Conference on Large Hadron Collider Physics - LHCP2019

20-25 May, 2019

Puebla, Mexico 


\section{Introduction}

Science outreach needs to be seen as an integral part of the scientific process that benefits both the scientists reaching out and those they reach out to, meaning society as a whole. Being intertwined with, yet different from science education, science communication and science policy making, science outreach is a major way of motivating the next generation of scientists and nurturing support for science in general and the field of particle physics in particular. It is also a principle instrument for passing on the accumulated know-how, experience and expertise gained through science and research to the public, which is especially important for publicly funded research. The public is, in general, curious. However, not all have our enthusiasm for fundamental research, nor perhaps an appreciation for how that research can have important effects on everyone's lives. It is thus necessary that we not only communicate our results, but that we make an effort to spark interest by sharing our excitement for the field and our enthusiasm for research in general. This is necessary, not only to provide the public the feedback for its fundamental support, but also to help prepare the general public for future projects. Doing good science outreach not only helps to increase science literacy and correct misconceptions about science and scientists, but also puts the scientist back into the public and improves links between scientists and society. Science outreach should also be as inclusive as possible and address also those that have less access to scientific research, in particular young people from groups poorly represented in the scientific field. For the individual scientist, the gain - besides the general benefits of a broader support of our field - is to get drawn out of the comfort zone and think about its own work in a bigger picture, and to improve - amongst other things - its teaching and communication skills.

\section{IPPOG}

So where does IPPOG - the International Particle Physics Outreach Group come in? IPPOG [1] is a network of scientists, science educators and communication specialists working across the globe in science education and public outreach for particle physics, the science of matter, energy, space and time. IPPOG brings new discoveries in this exciting field to young people and conveys to the public that the beauty of nature is indeed becoming understandable from the interactions of its most fundamental parts - the elementary particles. We contribute to global efforts in strengthening cultural awareness, understanding and support of particle physics and related sciences and in developing the next generation of researchers. More specifically, IPPOG's purpose is to raise standards of public outreach and science education efforts. In other words, IPPOG has been set up to support laboratories, collaborations, institutes and individuals in doing particlephysics outreach and education.

IPPOG emerged from EPPOG - the European Particle Physics Outreach Group in 2011 after the successful start-up of the Large Hadron Collider to reflect its true international stature, while EPPOG was formed already in 1997 by the European Committee for Future Accelerators (ECFA) and the High Energy Particle Physics Board of the European Physical Society (EPS-HEPP Board). In December 2016 IPPOG was formally converted into a collaboration that currently comprises 30 members: 24 countries, 5 experiments and CERN as an international laboratory; and is open to 
any country, international collaboration or international laboratory with an active particle physics research programme to join.

IPPOG member representatives and interested individuals are invited twice a year to collaboration meetings held at CERN or hosted by any of the members, to present, discuss and develop new ideas and approaches to educate, communicate and promote particle physics and science in general. IPPOG maintains various working groups e.g. to develop new exhibits, exhibitions and outreach-event ideas; to extend the reach of IPPOG-driven outreach programmes; or to find means to communicate the sometimes hard-to-grasp details/aspects of particle physics to audiences of all ages and educational levels. In addition IPPOG aims to be a provider/mediator for everyone interested to do particle-physics outreach and operates an extensive and searchable database of educational and outreach material [2] to ease the initial process and prevent people from reinventing the wheel. Last but not least, IPPOG provides support for individuals associated to any of its members who seek assistance implementing particle physics education and outreach projects with a potential to reach new, large and diverse audiences. This has included public events at art, music or science festivals or held in conjunction with major conferences.

\section{IPPOG Activities}

Probably the most prestigious and well-known project coordinated by IPPOG are the International Masterclasses on Particle Physics (IMC) [3], an annual programme organised by TU Dresden [4] and QuarkNet [5] Notre Dame, involving more than 13'000 high school students from more than 50 countries world-wide each year. Alone this year, with the help of almost 70 volunteer hosts at CERN, Fermilab and TRIUMF, this programme organised 323 masterclasses. Each of these is a one-day event that includes lectures from active scientists, a hands-on session with measurements using real experimental data and an international video conference held between classrooms from around the globe. The video conferences give students a chance to discuss results, compare and combine their measurements, and get a taste of real international scientific collaboration. The hands-on sessions use data provided by the ALICE [6], ATLAS [7], CMS [8], LHCb [9] and MINERvA [10] collaborations.

Over the past years, IPPOG has supported numerous other outreach projects to promote particle physics to a wide and diverse audience such as 'Particles4U' [11], 'Girls, do Physics' [12], as well as local events accompanying science conferences or festivals. Particles $4 U$ was a competition run by IPPOG in late 2017, funded by an award by the European Physical Society, challenging young students, teachers and classrooms to develop projects describing to the public the relevance of particle physics in everyday life. The project website and application forms were translated into 18 different languages to be as inclusive as possible and IPPOG received 77 legitimate responses from 15 countries world-wide. Girls, do Physics was an Instagram campaign to inspire girls to become interested in and study physics and related STEM subjects and break the stereotype that physics is more suitable for boys. IPPOG invited girls to create their you own physics project or scientific experiment, take a picture or make a video showing the project. 


\section{IPPOG and EPPSU}

IPPOG is continuously growing and more countries and laboratories have expressed interest in joining the effort in supporting particle-physics outreach world-wide. IPPOG is working on an improved resource database, has provided input [14] to the ongoing European Particle Physics Strategic Update (EPPSU) [13]. EPPSU, initiated by the CERN Council, provides a prioritisation of European ambitions in advancing particle-physics science, also taking into account the worldwide particle-physics landscape and developments in related fields. By Spring 2020, a recommendation will be established that shall recommend the most important projects in the quest for exploring the basic building blocks of matter, the structure of time and space, and with it the origins of our Universe. Large-scale facilities will be required to make significant steps in deepening our understanding and for opening new paths for future research. The duration of the planning, construction, commissioning, and operation stages of such large-scale facilities, regardless of the prioritisation and decisions that will be made among the candidate options of today, will span many decades. The execution of these programmes thus relies heavily on support from key stakeholders and decision makers, including politicians, funding agencies and, underlying all, the public. Furthermore, the success of such large-scale projects depends heavily on the talents of a skilled, enthusiastic, and diverse new generation of physicists, engineers, and developers. This is where IPPOG is particularly well-suited to play its role as a strategic pillar in the strategy update. IPPOG is laying down the ground work to enhance public sentiment favouring fundamental scientific research. Young audiences, which are a main target via the particle physics masterclasses programme, 'Particles4U', or 'Girls, do Physics', will be more inclined to become the next generation of scientists, engineers and technicians. Outreach is indeed not just to be done to explain scientific results to the public, but it is a necessity for society to appreciate the scientific process and evidence-based reasoning as cultural elements of society and therefore appreciate the necessity for fundamental research to take its next big steps towards a deeper understanding of the elementary building blocks of matter and all there is in the Universe.

\section{References}

[1] IPPOG Collaboration Website; http://ippog.org/

[2] IPPOG Resource Database; http://ippog.org/resources

[3] IPPOG Particle Physics Masterclasses; https://physicsmasterclasses.org/

[4] TU Dresden - Institute of Nuclear and Particle Physics; http://iktp.tu-dresden.de/

[5] QuarkNet; https://quarknet.org/

[6] The ALICE Collaboration; http://alice.web.cern.ch/

[7] The ATLAS Collaboration; https://atlas.cern/

[8] The CMS Collaboration; https://cms.cern/

[9] The LHCb Collaboration; http://lhcb-public.web.cern.ch/

[10] The MINERvA Collaboration; https://minerva.fnal.gov/

[11] Particles4U; http://ippog.org/particles4u

[12] Girls, do Physics; http://ippog.org/girlsdophysics

[13] EPPSU; http://europeanstrategyupdate.web.cern.ch/

[14] IPPOG EPPSU Input; https://indico.cern.ch/event/765096/contributions/3295747/ 